\title{
PEALD-TiN based Thin Films for High Performance Metallic Bipolar Plates of PEMFCs
}

\author{
Se-Hun Kwon', Eun-Young Yun', Han-Bo-Ram Lee ${ }^{3}$ \\ ${ }^{1,2}$ School of Materials Science and Engineering/Pusan National University \\ 30 Jangjeon-dong Geumjeong-gu, Busan, Republic of Korea \\ sehun@pusan.ac.kr; yey0620@naver.com \\ ${ }^{3}$ Department of Materials Science and Engineering/Incheon National University \\ 119 Academy-ro, Yeonsu-gu, Incheon 22012, Republic of Korea \\ hbrlee@inu.ac.kr
}

\section{Extended Abstract}

In the field of metallic bipolar plates for polymer electrolyte membrane fuel cells (PEMFCs), the development of protective coating that exhibit high electrical conductivity and corrosion resistance, as well as low interfacial contact resistance (ICR), is an essential scientific and engineering goal because these properties mainly determine the lifetime and performance of PEMFCs [1]. Furthermore, these protective coatings are a key technology in the mass-production of fuelcell-based automobiles. To meet the DOE's critical requirements for bipolar plates, extensive effort has been undertaken to develop adequate coating materials [2] to improve the electrical and corrosion properties of metallic bipolar plates, such as stainless steel 316L (SS316L). Recent achievements in this area has been based on the good performance of Au and carbonbased coatings. However, those coating materials are almost impossible to be commercialized, due to the high material cost of $\mathrm{Au}$ and the insufficient electrical conductivity of carbon-based coatings, although there have been many efforts to improve the drawbacks. Accordingly, there have been extensive efforts to find alternate materials and processes for Au or carbonbased coatings. Up to now, various surface-coating technologies, such as CVD, PVD, electroplating, and arc ion plating have been investigated for this application [3]. However, the coatings created through those methods do not always achieve satisfactory performance, and are still in competition for the application of coatings to bipolar plates.

Therefore, in our work, we first report the plasma-enhanced atomic layer deposition (PEALD) of TiN-based thin films to improve the electrical and corrosion properties of stainless steel 316L (SS316L) for this application. Due to the inherent merits of PEALD, such as precise thickness controllability, good step coverage, uniformity, and a pinhole-free nature, we can realize ultrathin and high performance TiN protective films, that can meet the strict requirements of the DOE such as sufficiently lower corrosion current density than $10^{-6} \mathrm{~A} / \mathrm{cm}^{2}$ and lower ICR values than $20 \mathrm{~m} \Omega \mathrm{cm}^{2}$, using an optimized PEALD process. Notably, it is based on low-cost titanium nitride (TiN) material with an ultrathin $(25 \sim 67 \mathrm{~nm})$ thicknesses. In addition to its performance, we carefully investigate which types of PEALD (based on halide and metal-organic precursors) are most appropriate for this application. Through careful and systemic study, we have discussed the reasons for the superior performance of TDMAT-based PEALD. According to our observation, the formation of ultrathin amorphous interfacial layers when using a TDMAT precursor was a key factor to ensure the performance.

\section{References}

[1] H. Tawfik, Y. Hung, and D. Mahajan, "Metal bipolar plates for PEM fuel cell - a review," J. Power Sources, vol. 163, pp. 755-767, 2007.

[2] A. Pozio, F. Zaza, A. Masci, and R. F. Silva, "Bipolar plate materials for PEMFCs: a conductivity and stability study," J. Power Sources, vol. 179, pp. 631-639, 2008.

[3] R. A. Antunes, M. C. L. Oliveira, G. Ett, and V. Ett, "B Corrosion of metal bipolar plates for PEM fuel cells: A review," Int. J. Hydrogen Energy, vol. 35, pp. 3632-3647, 2010. 